\title{
Stability of Equilibrium Points of Fractional Difference Equations with Stochastic Perturbations
}

\author{
Beatrice Paternoster ${ }^{1}$ and Leonid Shaikhet ${ }^{2}$ \\ ${ }^{1}$ Dipartimento di Matematica e Informatica, Universita di Salerno, via Ponte Don Melillo, \\ 84084 Fisciano $(\mathrm{Sa})$, Italy \\ ${ }^{2}$ Department of Higher Mathematics, Donetsk State University of Management, \\ 163 a Chelyuskintsev street, 83015 Donetsk, Ukraine
}

Correspondence should be addressed to Leonid Shaikhet, leonid.shaikhet@usa.net

Received 6 December 2007; Accepted 9 May 2008

Recommended by Jianshe Yu

It is supposed that the fractional difference equation $x_{n+1}=\left(\mu+\sum_{j=0}^{k} a_{j} x_{n-j}\right) /\left(\lambda+\sum_{j=0}^{k} b_{j} x_{n-j}\right)$, $n=0,1, \ldots$, has an equilibrium point $\hat{x}$ and is exposed to additive stochastic perturbations type of $\sigma\left(x_{n}-\widehat{x}\right) \xi_{n+1}$ that are directly proportional to the deviation of the system state $x_{n}$ from the equilibrium point $\widehat{x}$. It is shown that known results in the theory of stability of stochastic difference equations that were obtained via V. Kolmanovskii and L. Shaikhet general method of Lyapunov functionals construction can be successfully used for getting of sufficient conditions for stability in probability of equilibrium points of the considered stochastic fractional difference equation. Numerous graphical illustrations of stability regions and trajectories of solutions are plotted.

Copyright (C) 2008 B. Paternoster and L. Shaikhet. This is an open access article distributed under the Creative Commons Attribution License, which permits unrestricted use, distribution, and reproduction in any medium, provided the original work is properly cited.

\section{Introduction-Equilibrium points}

Recently, there is a very large interest in studying the behavior of solutions of nonlinear difference equations, in particular, fractional difference equations [1-38]. This interest really is so large that a necessity appears to get some generalized results.

Here, the stability of equilibrium points of the fractional difference equation

$$
x_{n+1}=\frac{\mu+\sum_{j=0}^{k} a_{j} x_{n-j}}{\lambda+\sum_{j=0}^{k} b_{j} x_{n-j}}, \quad n \in Z=\{0,1, \ldots\},
$$

with the initial condition

$$
x_{j}=\phi_{j}, \quad j \in Z_{0}=\{-k,-k+1, \ldots, 0\},
$$


is investigated. Here $\mu, \lambda, a_{j}, b_{j}, j=0, \ldots, k$ are known constants. Equation (1.1) generalizes a lot of different particular cases that are considered in $[1-8,16,18-20,22-24,32,35,37]$.

Put

$$
A_{j}=\sum_{l=j}^{k} a_{j}, \quad B_{j}=\sum_{l=j}^{k} b_{j}, \quad j=0,1, \ldots, k, A=A_{0}, B=B_{0},
$$

and suppose that (1.1) has some point of equilibrium $\widehat{x}$ (not necessary a positive one). Then by assumption

$$
\lambda+B \widehat{x} \neq 0
$$

the equilibrium point $\widehat{x}$ is defined by the algebraic equation

$$
\widehat{x}=\frac{\mu+A \widehat{x}}{\lambda+B \widehat{x}} .
$$

By condition (1.4), equation (1.5) can be transformed to the form

$$
B \widehat{x}^{2}-(A-\lambda) \widehat{x}-\mu=0 .
$$

It is clear that if

$$
(A-\lambda)^{2}+4 B \mu>0
$$

then (1.1) has two points of equilibrium

$$
\begin{aligned}
& \widehat{x}_{1}=\frac{A-\lambda+\sqrt{(A-\lambda)^{2}+4 B \mu}}{2 B}, \\
& \widehat{x}_{2}=\frac{A-\lambda-\sqrt{(A-\lambda)^{2}+4 B \mu}}{2 B} .
\end{aligned}
$$

If

$$
(A-\lambda)^{2}+4 B \mu=0,
$$

then (1.1) has only one point of equilibrium

$$
\widehat{x}=\frac{A-\lambda}{2 B} \text {. }
$$

And at last if

$$
(A-\lambda)^{2}+4 B \mu<0
$$

then (1.1) has not equilibrium points.

Remark 1.1. Consider the case $\mu=0, B \neq 0$. From (1.5) we obtain the following. If $\lambda \neq 0$ and $A \neq \lambda$, then (1.1) has two points of equilibrium:

$$
\widehat{x}_{1}=\frac{A-\lambda}{B}, \quad \widehat{x}_{2}=0
$$

If $\lambda \neq 0$ and $A=\lambda$, then (1.1) has only one point of equilibrium: $\widehat{x}=0$. If $\lambda=0$, then (1.1) has only one point of equilibrium: $\widehat{x}=A / B$.

Remark 1.2. Consider the case $\mu=B=0, \lambda \neq 0$. If $A \neq \lambda$, then (1.1) has only one point of equilibrium: $\widehat{x}=0$. If $A=\lambda$, then each solution $\widehat{x}=$ const is an equilibrium point of (1.1). 


\section{Stochastic perturbations, centering, and linearization-Definitions and auxiliary statements}

Let $\{\Omega, \mathfrak{F}, \mathbf{P}\}$ be a probability space and let $\left\{\mathfrak{F}_{n}, n \in Z\right\}$ be a nondecreasing family of sub- $\sigma$ algebras of $\mathfrak{F}$, that is, $\mathfrak{F}_{n_{1}} \subset \mathfrak{F}_{n_{2}}$ for $n_{1}<n_{2}$, let $\mathbf{E}$ be the expectation, let $\xi_{n}, n \in Z$, be a sequence of $\mathfrak{F}_{n}$-adapted mutually independent random variables such that $\mathbf{E} \xi_{n}=0, \mathbf{E} \xi_{n}^{2}=1$.

As it was proposed in $[39,40]$ and used later in [41-43] we will suppose that (1.1) is exposed to stochastic perturbations $\xi_{n}$ which are directly proportional to the deviation of the state $x_{n}$ of system (1.1) from the equilibrium point $\widehat{x}$. So, (1.1) takes the form

$$
x_{n+1}=\frac{\mu+\sum_{j=0}^{k} a_{j} x_{n-j}}{\lambda+\sum_{j=0}^{k} b_{j} x_{n-j}}+\sigma\left(x_{n}-\widehat{x}\right) \xi_{n+1} .
$$

Note that the equilibrium point $\hat{x}$ of (1.1) is also the equilibrium point of (2.1).

Putting $y_{n}=x_{n}-\widehat{x}$ we will center (2.1) in the neighborhood of the point of equilibrium $\widehat{x}$. From (2.1) it follows that

$$
y_{n+1}=\frac{\sum_{j=0}^{k}\left(a_{j}-b_{j} \widehat{x}\right) y_{n-j}}{\lambda+B \widehat{x}+\sum_{j=0}^{k} b_{j} y_{n-j}}+\sigma y_{n} \xi_{n+1} .
$$

It is clear that the stability of the trivial solution of (2.2) is equivalent to the stability of the equilibrium point of (2.1).

Together with nonlinear equation (2.2) we will consider and its linear part

$$
z_{n+1}=\sum_{j=0}^{k} \gamma_{j} z_{n-j}+\sigma z_{n} \xi_{n+1}, \quad \gamma_{j}=\frac{a_{j}-b_{j} \widehat{x}}{\lambda+B \widehat{x}}
$$

Two following definitions for stability are used below.

Definition 2.1. The trivial solution of (2.2) is called stable in probability if for any $\epsilon_{1}>0$ and $\epsilon_{2}>$ 0 there exists $\delta>0$ such that the solution $y_{n}=y_{n}(\phi)$ satisfies the condition $\mathbf{P}\left\{\sup _{n \in Z}\left|y_{n}(\phi)\right|>\right.$ $\left.\epsilon_{1}\right\}<\epsilon_{2}$ for any initial function $\phi$ such that $\mathbf{P}\left\{\sup _{j \in Z_{0}}\left|\phi_{j}\right| \leq \delta\right\}=1$.

Definition 2.2. The trivial solution of (2.3) is called mean square stable if for any $\epsilon>0$ there exists $\delta>0$ such that the solution $z_{n}=z_{n}(\phi)$ satisfies the condition $\mathbf{E}\left|z_{n}(\phi)\right|^{2}<\epsilon$ for any initial function $\phi$ such that $\sup _{j \in Z_{0}} \mathbf{E}\left|\phi_{j}\right|^{2}<\delta$. If, besides, $\lim _{n \rightarrow \infty} \mathbf{E}\left|z_{n}(\phi)\right|^{2}=0$, for any initial function $\phi$, then the trivial solution of (2.3) is called asymptotically mean square stable.

The following method for stability investigation is used below. Conditions for asymptotic mean square stability of the trivial solution of constructed linear equation (2.3) were obtained via V. Kolmanovskii and L. Shaikhet general method of Lyapunov functionals construction [44-46]. Since the order of nonlinearity of (2.2) is more than 1, then obtained stability conditions at the same time are [47-49] conditions for stability in the probability of the trivial solution of nonlinear equation (2.2) and therefore for stability in probability of the equilibrium point of (2.1). 
Lemma 2.3. (see [44]). If

$$
\sum_{j=0}^{k}\left|\gamma_{j}\right|<\sqrt{1-\sigma^{2}}
$$

then the trivial solution of (2.3) is asymptotically mean square stable.

Put

$$
\beta=\sum_{j=0}^{k} r_{j}, \quad \alpha=\sum_{j=1}^{k}\left|G_{j}\right|, \quad G_{j}=\sum_{l=j}^{k} \gamma_{l} .
$$

Lemma 2.4. (see [44]). If

$$
\beta^{2}+2 \alpha|1-\beta|+\sigma^{2}<1
$$

then the trivial solution of (2.3) is asymptotically mean square stable.

Consider also the necessary and sufficient condition for asymptotic mean square stability of the trivial solution of (2.3).

Let $U$ and $\Gamma$ be two square matrices of dimension $k+1$ such that $U=\left\|u_{i j}\right\|$ has all zero elements except for $u_{k+1, k+1}=1$ and

$$
\Gamma=\left(\begin{array}{cccccc}
0 & 1 & 0 & \cdots & 0 & 0 \\
0 & 0 & 1 & \cdots & 0 & 0 \\
\cdots & \cdots & \cdots & \cdots & \cdots & \cdots \\
0 & 0 & 0 & \cdots & 0 & 1 \\
\gamma_{k} & \gamma_{k-1} & \gamma_{k-2} & \cdots & \gamma_{1} & \gamma_{0}
\end{array}\right)
$$

Lemma 2.5 ([46]). Let the matrix equation

$$
\Gamma^{\prime} D \Gamma-D=-U
$$

has a positively semidefinite solution $D$ with $d_{k+1, k+1}>0$. Then the trivial solution of (2.3) is asymptotically mean square stable if and only if

$$
\sigma^{2} d_{k+1, k+1}<1 .
$$

Corollary 2.6. For $k=1$ condition (2.9) takes the form

$$
\begin{gathered}
\left|\gamma_{1}\right|<1, \quad\left|\gamma_{0}\right|<1-\gamma_{1}, \\
\sigma^{2}<d_{22}^{-1}=\frac{\left(1+\gamma_{1}\right)\left(\left(1-\gamma_{1}\right)^{2}-\gamma_{0}^{2}\right)}{1-\gamma_{1}} .
\end{gathered}
$$

If, in particular, $\sigma=0$, then condition (2.10) is the necessary and sufficient condition for asymptotic mean square stability of the trivial solution of (2.3) for $k=1$.

Remark 2.7. Put $\sigma=0$. If $\beta=1$, then the trivial solution of (2.3) can be stable (e.g., $z_{n+1}=z_{n}$ or $z_{n+1}=0.5\left(z_{n}+z_{n-1}\right)$ ), unstable (e.g., $z_{n+1}=2 z_{n}-z_{n-1}$ ) but cannot be asymptotically stable. 
Really, it is easy to see that if $\beta \geq 1$ (in particular, $\beta=1$ ), then sufficient conditions (2.4) and (2.6) do not hold. Moreover, necessary and sufficient (for $k=1$ ) condition (2.10) does not hold too since if (2.10) holds, then we obtain a contradiction

$$
1 \leq \beta=\gamma_{0}+\gamma_{1} \leq\left|\gamma_{0}\right|+\gamma_{1}<1
$$

Remark 2.8. As it follows from results of [47-49] the conditions of Lemmas 2.3, 2.4, 2.5 at the same time are conditions for stability in probability of the equilibrium point of (2.1).

\section{Stability of equilibrium points}

From conditions (2.4), (2.6) it follows that $|\beta|<1$. Let us check if this condition can be true for each equilibrium point.

Suppose at first that condition (1.7) holds. Then (2.1) has two points of equilibrium $\widehat{x}_{1}$ and $\widehat{x}_{2}$ defined by (1.8) and (1.9) accordingly. Putting $S=\sqrt{(A-\lambda)^{2}+4 B \mu}$ via (2.5), (2.3), (1.3), we obtain that corresponding $\beta_{1}$ and $\beta_{2}$ are

$$
\begin{aligned}
& \beta_{1}=\frac{A-B \widehat{x}_{1}}{\lambda+B \widehat{x}_{1}}=\frac{A-(1 / 2)(A-\lambda+S)}{\lambda+(1 / 2)(A-\lambda+S)}=\frac{A+\lambda-S}{A+\lambda+S} \\
& \beta_{2}=\frac{A-B \widehat{x}_{2}}{\lambda+B \widehat{x}_{2}}=\frac{A-(1 / 2)(A-\lambda-S)}{\ell+(1 / 2)(A-\lambda-S)}=\frac{A+\lambda+S}{A+\lambda-S} .
\end{aligned}
$$

So, $\beta_{1} \beta_{2}=1$. It means that the condition $|\beta|<1$ holds only for one from the equilibrium points $\widehat{x}_{1}$ and $\widehat{x}_{2}$. Namely, if $A+\lambda>0$, then $\left|\beta_{1}\right|<1$; if $A+\lambda<0$, then $\left|\beta_{2}\right|<1$; if $A+\lambda=0$, then $\beta_{1}=\beta_{2}=-1$. In particular, if $\mu=0$, then via Remark 1.1 and (2.3) we have $\beta_{1}=\lambda A^{-1}$, $\beta_{2}=\lambda^{-1} A$. Therefore, $\left|\beta_{1}\right|<1$ if $|\lambda|<|A|,\left|\beta_{2}\right|<1$ if $|\lambda|>|A|,\left|\beta_{1}\right|=\left|\beta_{2}\right|=1$ if $|\lambda|=|A|$.

So, via Remark 2.7, we obtain that equilibrium points $\widehat{x}_{1}$ and $\widehat{x}_{2}$ can be stable concurrently only if corresponding $\beta_{1}$ and $\beta_{2}$ are negative concurrently.

Suppose now that condition (1.10) holds. Then (2.1) has only one point of equilibrium (1.11). From (2.5), (2.3), (1.3), (1.11) it follows that corresponding $\beta$ equals

$$
\beta=\frac{A-B \widehat{x}}{\lambda+B \widehat{x}}=\frac{A-(1 / 2)(A-\lambda)}{\lambda+(1 / 2)(A-\lambda)}=\frac{A+\lambda}{\lambda+A}=1
$$

As it follows from Remark 2.7 this point of equilibrium cannot be asymptotically stable.

Corollary 3.1. Let $\widehat{x}$ be an equilibrium point of (2.1) such that

$$
\sum_{j=0}^{k}\left|a_{j}-b_{j} \hat{x}\right|<|\lambda+B \hat{x}| \sqrt{1-\sigma^{2}}, \quad \sigma^{2}<1
$$

Then the equilibrium point $\widehat{x}$ is stable in probability.

The proof follows from (2.3), Lemma 2.3, and Remark 2.8. 
Corollary 3.2. Let $\widehat{x}$ be an equilibrium point of (2.1) such that

$$
\begin{gathered}
|A-B \hat{x}|<|\lambda+B \widehat{x}|, \\
2 \sum_{j=1}^{k}\left|A_{j}-B_{j} \hat{x}\right|<|\lambda+A|-\sigma^{2} \frac{(\lambda+B \widehat{x})^{2}}{|\lambda-A+2 B \hat{x}|} .
\end{gathered}
$$

Then the equilibrium point $\widehat{x}$ is stable in probability.

Proof. Via (1.3), (2.3), (2.5) we have

$$
\alpha=|\lambda+B \widehat{x}|^{-1} \sum_{j=1}^{k}\left|A_{j}-B_{j} \widehat{x}\right|, \quad \beta=\frac{A-B \widehat{x}}{\lambda+B \widehat{x}} .
$$

Rewrite (2.6) in the form

$$
2 \alpha<1+\beta-\frac{\sigma^{2}}{1-\beta}, \quad|\beta|<1,
$$

and show that it holds. From (3.4) it follows that $|\beta|<1$. Via $|\beta|<1$ we have

$$
\begin{aligned}
& 1+\beta=1+\frac{A-B \widehat{x}}{\lambda+B \widehat{x}}=\frac{\lambda+A}{\lambda+B \widehat{x}}>0, \\
& 1-\beta=1-\frac{A-B \widehat{x}}{\lambda+B \widehat{x}}=\frac{\lambda-A+2 B \widehat{x}}{\lambda+B \widehat{x}}>0 .
\end{aligned}
$$

So,

$$
2 \sum_{j=1}^{k}\left|A_{j}-B_{j} \widehat{x}\right|<|\lambda+B \widehat{x}|\left(\frac{\lambda+A}{\lambda+B \widehat{x}}-\sigma^{2} \frac{\lambda+B \widehat{x}}{\lambda-A+2 B \widehat{x}}\right)=|\lambda+A|-\sigma^{2} \frac{(\lambda+B \widehat{x})^{2}}{|\lambda-A+2 B \hat{x}|} .
$$

It means that the condition of Lemma 2.4 holds. Via Remark 2.8 the proof is completed.

Corollary 3.3. An equilibrium point $\widehat{x}$ of the equation

$$
x_{n+1}=\frac{\mu+a_{0} x_{n}+a_{1} x_{n-1}}{\lambda+b_{0} x_{n}+b_{1} x_{n-1}}+\sigma\left(x_{n}-\widehat{x}\right) \xi_{n+1}
$$

is stable in probability if and only if

$$
\begin{gathered}
\left|a_{1}-b_{1} \hat{x}\right|<|\lambda+B \hat{x}|, \\
\left|a_{0}-b_{0} \widehat{x}\right|<\left(\lambda-a_{1}+\left(b_{0}+2 b_{1}\right) \hat{x}\right) \operatorname{sign}(\lambda+B \widehat{x}), \\
\sigma^{2}<\frac{\left(\lambda+a_{1}+b_{0} \hat{x}\right)\left(\lambda+a_{0}-a_{1}+2 b_{1} \widehat{x}\right)(\lambda-A+2 B \widehat{x})}{\left(\lambda-a_{1}+\left(b_{0}+2 b_{1}\right) \hat{x}\right)(\lambda+B \hat{x})^{2}} .
\end{gathered}
$$

The proof follows from (2.3), (2.10), (2.11). 


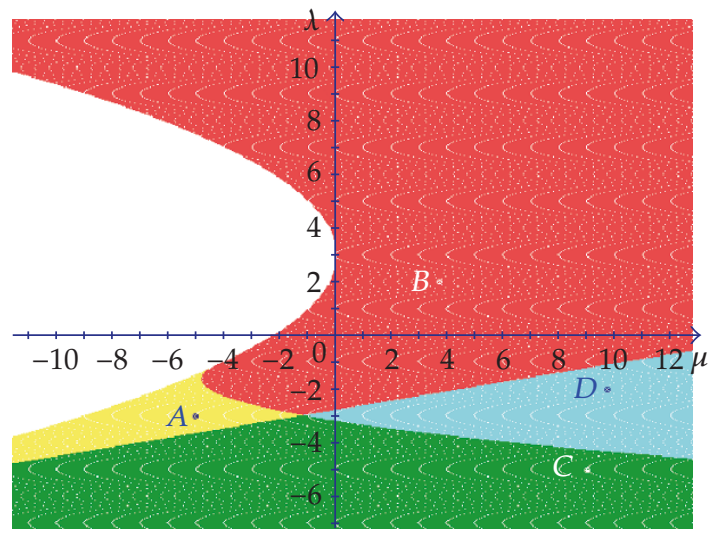

Figure 1: Stability regions, $\sigma^{2}=0$.

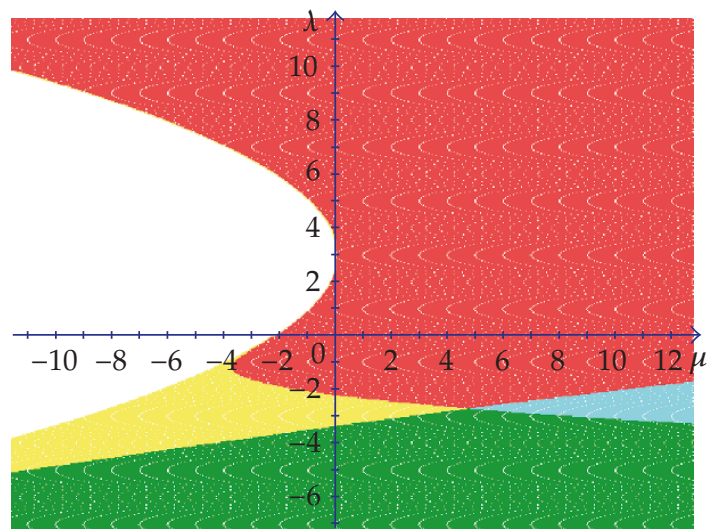

Figure 2: Stability regions, $\sigma^{2}=0.3$.

\section{Examples}

Example 4.1. Consider (3.10) with $a_{0}=2.9, a_{1}=0.1, b_{0}=b_{1}=0.5$. From (1.3) and (1.7)-(1.9) it follows that $A=3, B=1$ and for any fixed $\mu$ and $\lambda$ such that $\mu>-(1 / 4)(3-\lambda)^{2}$ equation (3.10) has two points of equilibrium

$$
\widehat{x}_{1}=\frac{1}{2}\left(3-\lambda+\sqrt{(3-\lambda)^{2}+4 \mu}\right), \quad \widehat{x}_{2}=\frac{1}{2}\left(3-\lambda-\sqrt{(3-\lambda)^{2}+4 \mu}\right) .
$$

In Figure 1, the region where the points of equilibrium are absent (white region), the region where both points of equilibrium $\widehat{x}_{1}$ and $\widehat{x}_{2}$ are there but unstable (yellow region), the region where the point of equilibrium $\widehat{x}_{1}$ is stable only (red region), the region where the point of equilibrium $\widehat{x}_{2}$ is stable only (green region), and the region where both points of equilibrium $\widehat{x}_{1}$ and $\hat{x}_{2}$ are stable (cyan region) are shown in the space of $(\mu, \lambda)$. All regions are obtained via condition (3.11) for $\sigma^{2}=0$. In Figures 2, 3 one can see similar regions for $\sigma^{2}=0.3$ and $\sigma^{2}=0.8$, accordingly, that were obtained via conditions (3.11), (3.12). In Figure 4 it is shown that sufficient conditions (3.3) and (3.4), (3.5) are enough close to necessary and sufficient conditions (3.11), (3.12): inside of the region where the point of equilibrium $\widehat{x}_{1}$ is stable (red 


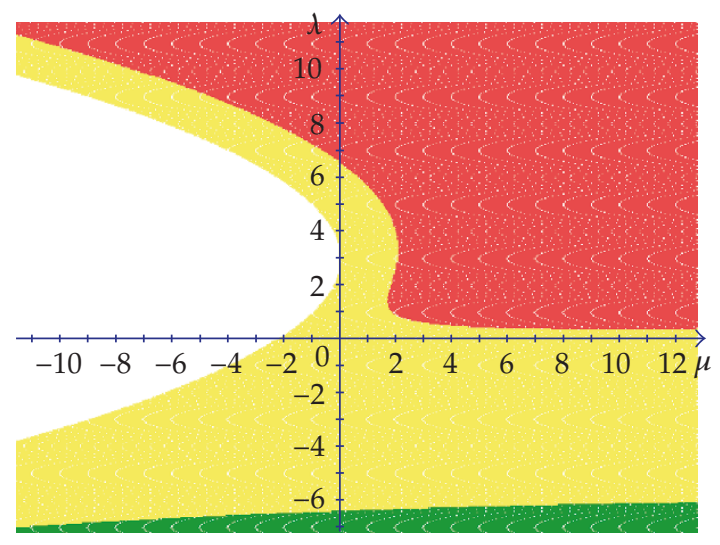

Figure 3: Stability regions, $\sigma^{2}=0.8$.

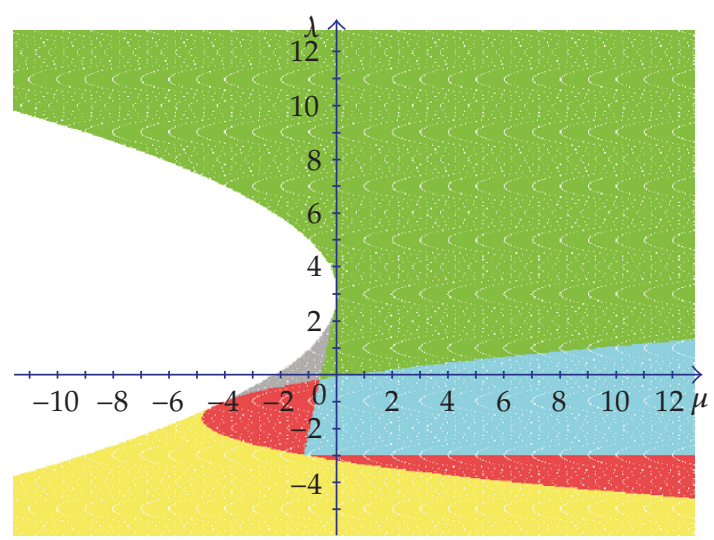

Figure 4: Stability regions, $\sigma^{2}=0$.

region) one can see the regions of stability of the point of equilibrium $\widehat{x}_{1}$ that were obtained by condition (3.3) (grey and green regions) and by conditions (3.4), (3.5) (cyan and green regions). Stability regions obtained via both sufficient conditions of stability (3.3) and (3.4), (3.5) give together almost whole stability region obtained via necessary and sufficient stability conditions (3.11), (3.12).

Consider now the behavior of solutions of (3.10) with $\sigma=0$ in the points $A, B, C, D$ of the space of $(\mu, \lambda)$ (Figure 1). In the point $A$ with $\mu=-5, \lambda=-3$ both equilibrium points $\widehat{x}_{1}=5$ and $\widehat{x}_{2}=1$ are unstable. In Figure 5 two trajectories of solutions of (3.10) are shown with the initial conditions $x_{-1}=5, x_{0}=4.95$, and $x_{-1}=0.999, x_{0}=1.0001$. In Figure 6 two trajectories of solutions of (3.10) with the initial conditions $x_{-1}=-3, x_{0}=13$, and $x_{-1}=-1.5$, $x_{0}=-1.500001$ are shown in the point $B$ with $\mu=3.75, \lambda=2$. One can see that the equilibrium point $\widehat{x}_{1}=2.5$ is stable and the equilibrium point $\widehat{x}_{2}=-1.5$ is unstable. In the point $C$ with $\mu=9$, $\lambda=-5$ the equilibrium point $\widehat{x}_{1}=9$ is unstable and the equilibrium point $\widehat{x}_{2}=-1$ is stable. Two corresponding trajectories of solutions are shown in Figure 7 with the initial conditions $x_{-1}=7$, $x_{0}=10$, and $x_{-1}=-8, x_{0}=8$. In the point $D$ with $\mu=9.75, \lambda=-2$ both equilibrium points $\hat{x}_{1}=6.5$ and $\widehat{x}_{2}=-1.5$ are stable. Two corresponding trajectories of solutions are shown in Figure 8 with the initial conditions $x_{-1}=2, x_{0}=12$, and $x_{-1}=-8, x_{0}=8$. As it was noted above in this case, corresponding $\beta_{1}$ and $\beta_{2}$ are negative: $\beta_{1}=-7 / 9$ and $\beta_{2}=-9 / 7$. 


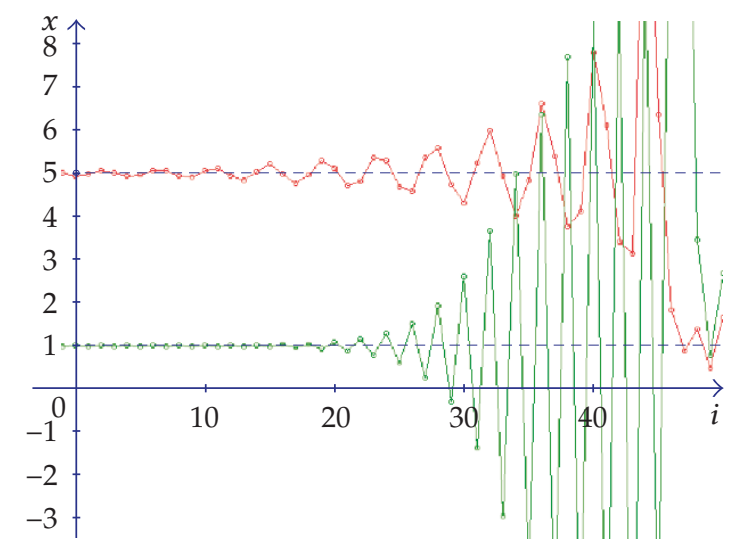

Figure 5: Unstable equilibrium points $\widehat{x}_{1}=5$ and $\widehat{x}_{2}=1$ for $\mu=-5, \lambda=-3$.

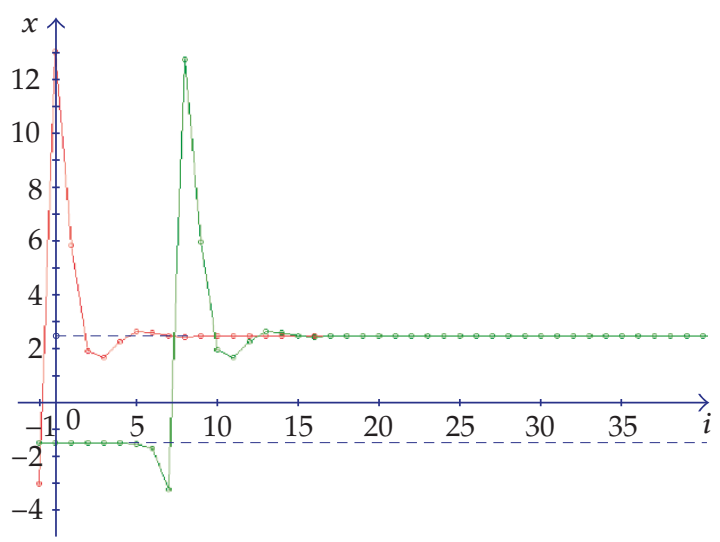

Figure 6: Stable equilibrium point $\widehat{x}_{1}=2.5$ and unstable $\widehat{x}_{2}=-1.5$ for $\mu=3.75, \lambda=2$.

Consider the difference equation

$$
x_{n+1}=p+q \frac{x_{n-m}}{x_{n-r}}+\sigma\left(x_{n}-\widehat{x}\right) \xi_{i+1} .
$$

Different particular cases of this equation were considered in $[2-5,16,22,23,37]$.

Equation (4.2) is a particular case of (2.1) with

$$
\begin{aligned}
a_{r}=p, & a_{m}=q, \quad a_{j}=0 \quad \text { if } j \neq r, j \neq m, \\
\mu=\lambda=0, & b_{r}=1, \quad b_{j}=0 \quad \text { if } j \neq r, \widehat{x}=p+q .
\end{aligned}
$$

Suppose firstly that $p+q \neq 0$ and consider two cases: (1) $m>r \geq 0$, (2) $r>m \geq 0$. In the first case,

$$
\begin{gathered}
A_{j}=p+q \text { if } j=0, \ldots, r, \quad A_{j}=q \text { if } j=r+1, \ldots, m, \\
B_{j}=1 \quad \text { if } j=0, \ldots, r, \quad B_{j}=0 \quad \text { if } j=r+1, \ldots, m .
\end{gathered}
$$

In the second case,

$$
A_{j}=p+q \quad \text { if } j=0, \ldots, m, \quad A_{j}=p \quad \text { if } j=m+1, \ldots, r, \quad B_{j}=1 \quad \text { if } j=0, \ldots, r .
$$




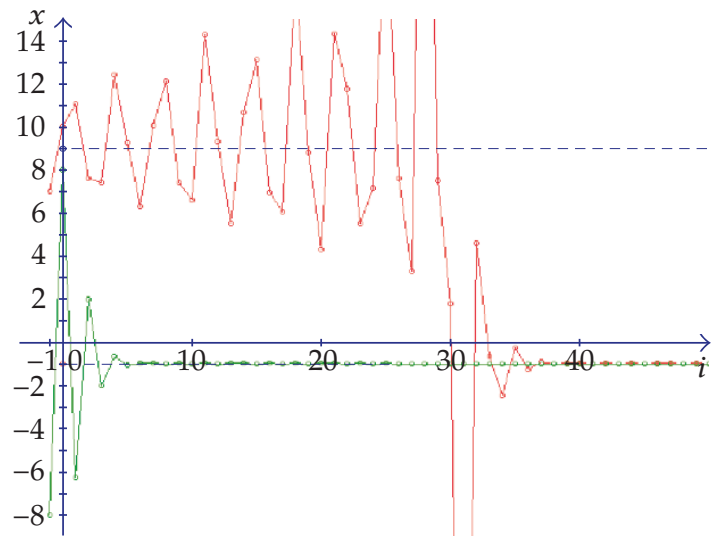

Figure 7: Unstable equilibrium point $\widehat{x}_{1}=9$ and stable $\widehat{x}_{2}=-1$ for $\mu=9, \lambda=-5$.

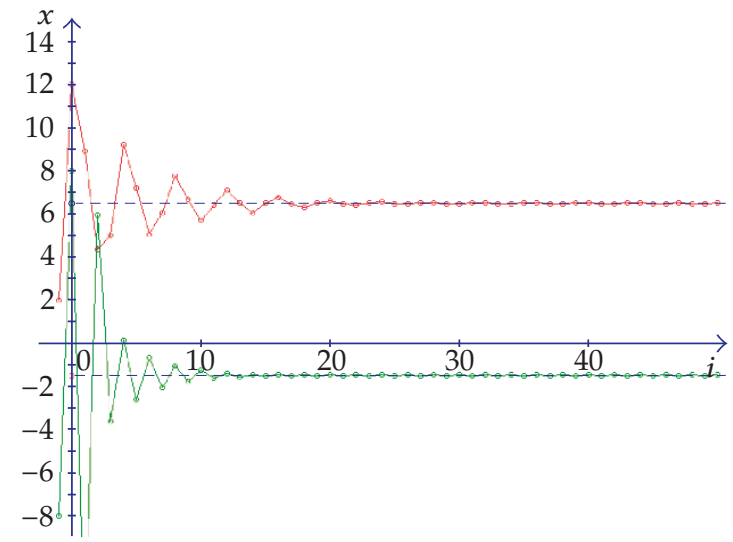

Figure 8: Stable equilibrium points $\widehat{x}_{1}=6.5$ and $\widehat{x}_{2}=-1.5$ for $\mu=9.75, \lambda=-2$.

In both cases, Corollary 3.1 gives stability condition in the form $2|q|<\sqrt{1-\sigma^{2}}|p+q|$ or

$$
p \in(-\infty,-q-\theta|q|) \cup(-q+\theta|q|, \infty)
$$

with

$$
\theta=\theta_{1}=\frac{2}{\sqrt{1-\sigma^{2}}}
$$

Corollary 3.2 in both cases gives stability condition in the form $2|q||m-r|<\left(1-\sigma^{2}\right)|p+q|$ or (4.6) with

$$
\theta=\theta_{2}=\frac{2|m-r|}{1-\sigma^{2}}
$$

Since $\theta_{2}>\theta_{1}$ then condition (4.6), (4.7) is better than (4.6), (4.8).

In the case $m=1, r=0$ Corollary 3.3 gives stability condition in the form

$$
|q|<|p+q|,|q|<p \operatorname{sign}(p+q), \quad \sigma^{2}<\frac{(p+2 q)(p-q)}{p(p+q)}
$$




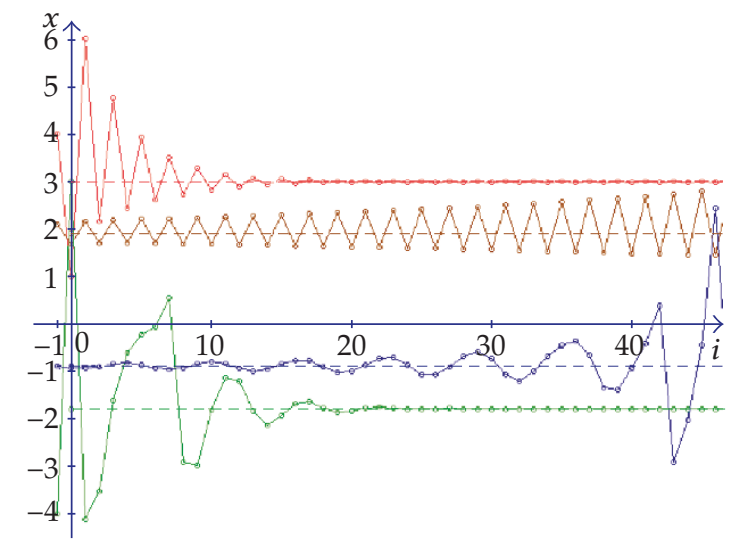

Figure 9: Stable equilibrium points $\widehat{x}=3$ and $\widehat{x}=-1.8$, unstable $\widehat{x}=1.93$ and $\widehat{x}=-0.9$.

or

$$
p \in\left(-\infty, \frac{1}{2}(-q-\theta|q|)\right) \cup\left(\frac{1}{2}(-q+\theta|q|), \infty\right), \quad \theta=\sqrt{\frac{9-\sigma^{2}}{1-\sigma^{2}}} .
$$

In particular, from (4.10) it follows that for $q=1, \sigma=0$ (this case was considered in $[3,23])$ the equilibrium point $\widehat{x}=p+1$ is stable if and only if $p \in(-\infty,-2) \cup(1, \infty)$. Note that in [3] for this case the condition $p>1$ only is obtained.

In Figure 9 four trajectories of solutions of (4.2) in the case $m=1, r=0, \sigma=0, q=1$ are shown: (1) $p=2, \widehat{x}=3, x_{-1}=4, x_{0}=1$ (red line, stable solution); (2) $p=0.93, \widehat{x}=1.93, x_{-1}=2.1$, $x_{0}=1.7$ (brown line, unstable solution); (3) $p=-1.9, \hat{x}=-0.9, x_{-1}=-0.89, x_{0}=-0.94$ (blue line, unstable solution); (4) $p=-2.8, \widehat{x}=-1.8, x_{-1}=-4, x_{0}=3$ (green line, stable solution).

In the case $r=1, m=0$, Corollary 3.3 gives stability condition in the form

$$
|q|<|p+q|,|q|<(p+2 q) \operatorname{sign}(p+q), \quad \sigma^{2}<\frac{p(p+3 q)}{(p+q)(p+2 q)}
$$

or

$$
p \in\left(-\infty, \frac{1}{2}(-3 q-\theta|q|)\right) \cup\left(\frac{1}{2}(-3 q+\theta|q|), \infty\right), \quad \theta=\sqrt{\frac{9-\sigma^{2}}{1-\sigma^{2}}}
$$

Example 4.2. For example, from (4.12) it follows that for $q=-1, \sigma=0$ (this case was considered in $[22,37])$, the equilibrium point $\widehat{x}=p-1$ is stable if and only if $p \in(-\infty, 0) \cup(3, \infty)$. In Figure 10 four trajectories of solutions of (4.2) in the case $r=1, m=0, \sigma=0, q=-1$ are shown: (1) $p=3.5, \widehat{x}=2.5, x_{-1}=3.5, x_{0}=1.5$ (red line, stable solution); (2) $p=2.2, \widehat{x}=1.2, x_{-1}=1.2$, $x_{0}=1.2001$ (brown line, unstable solution); (3) $p=0.3, \widehat{x}=-0.7, x_{-1}=-0.7, x_{0}=-0.705$ (blue line, unstable solution); (4) $p=-0.2, \widehat{x}=-1.2, x_{-1}=-2, x_{0}=-0.4$ (green line, stable solution).

Via simulation of a sequence of mutually independent random variables $\xi_{n}$ consider the behavior of the equilibrium point by stochastic perturbations. In Figure 11 one thousand trajectories are shown for $p=4, q=-1, \sigma=0.5, x_{-1}=3.5, x_{0}=2.5$. In this case, stability 


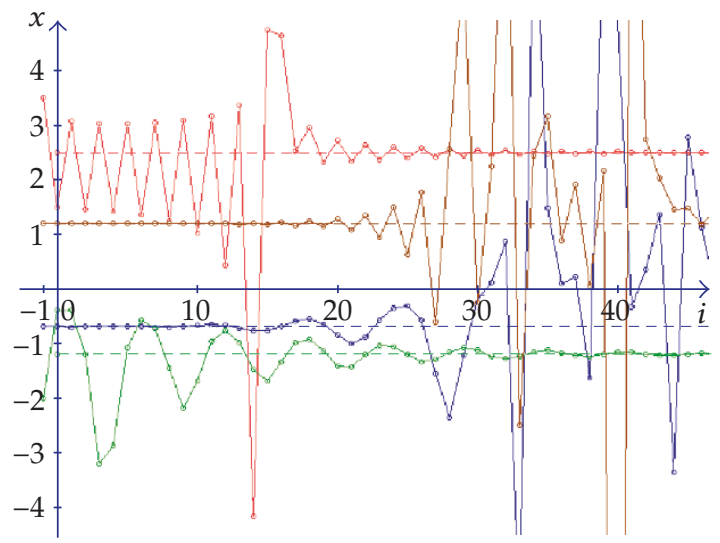

Figure 10: Stable equilibrium points $\widehat{x}=2.5$ and $\widehat{x}=-1.2$, unstable $\widehat{x}=1.2$ and $\widehat{x}=-0.7$.

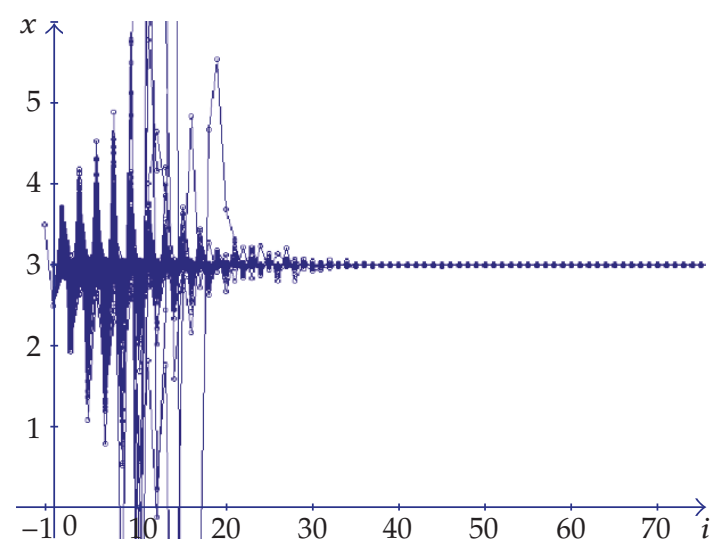

Figure 11: Stable equilibrium point $\widehat{x}=3$ for $p=4, q=-1, \sigma=0.5$.

condition (4.12) holds $(4 \in(-\infty,-0.2) \cup(3.2, \infty))$ and therefore the equilibrium point $\hat{x}=3$ is stable: all trajectories go to $\widehat{x}$. Putting $\sigma=0.9$, we obtain that stability condition (4.12) does not hold $(4 \notin(-\infty,-1.78) \cup(4.78, \infty))$. Therefore, the equilibrium point $\hat{x}=3$ is unstable: in Figure 12 one can see that 1000 trajectories fill the whole space.

Note also that if $p+q$ goes to zero all obtained stability conditions are violated. Therefore, by conditions $p+q=0$ the equilibrium point is unstable.

Example 4.3. Consider the equation

$$
x_{n+1}=\frac{\mu+a x_{n-1}}{\lambda+x_{n}}+\sigma\left(x_{n}-\widehat{x}\right) \xi_{n+1}
$$

(its particular cases were considered in $[18,19,35])$. Equation $(4.13)$ is a particular case of (2.1) with $k=1, a_{0}=b_{1}=0, a_{1}=a, b_{0}=1$. From (1.7)-(1.9) it follows that by condition $\mu>-(1 / 4)(a-\lambda)^{2}$ it has two equilibrium points

$$
\widehat{x}_{1}=\frac{a-\lambda+S}{2}, \quad \widehat{x}_{2}=\frac{a-\lambda-S}{2}, \quad S=\sqrt{(a-\lambda)^{2}+4 \mu} .
$$




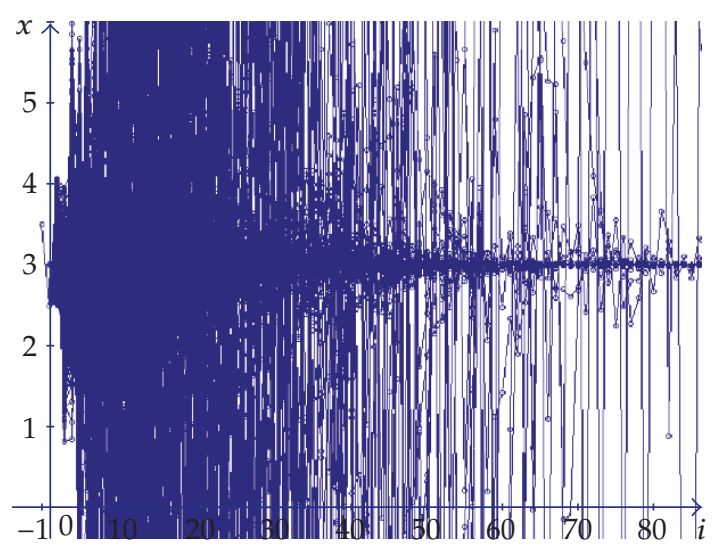

Figure 12: Unstable equilibrium point $\widehat{x}=3$ for $p=4, q=-1, \sigma=0.9$.

For equilibrium point $\widehat{x}$ sufficient conditions (3.3) and (3.4), (3.5) give

$$
\begin{gathered}
|\hat{x}|+|a|<|\lambda+\widehat{x}| \sqrt{1-\sigma^{2}}, \\
2|a|<|\lambda+a|-\sigma^{2} \frac{(\lambda+\widehat{x})^{2}}{|\lambda+2 \hat{x}-a|}, \quad|a-\hat{x}|<|\lambda+\widehat{x}| .
\end{gathered}
$$

From (3.11), (3.12) it follows that an equilibrium point $\widehat{x}$ of (4.13) is stable in probability if and only if

$$
\begin{gathered}
|\lambda+\hat{x}|>|a|, \quad|\widehat{x}|<(\lambda+\widehat{x}-a) \operatorname{sign}(\lambda+\widehat{x}), \\
\sigma^{2}<\frac{(\lambda+\widehat{x}+a)(\lambda-a)(\lambda+2 \widehat{x}-a)}{(\lambda+\widehat{x}-a)(\lambda+\widehat{x})^{2}} .
\end{gathered}
$$

For example, for $\widehat{x}=\widehat{x}_{1}$ from (4.15) we obtain

$$
\begin{gathered}
|a-\lambda+S|+2|a|<|a+\lambda+S| \sqrt{1-\sigma^{2}}, \\
2|a|<\mathcal{\lambda}+a-\sigma^{2} \frac{(\lambda+a+S)^{2}}{4 S}, \quad \lambda+a>0 .
\end{gathered}
$$

From (4.16) it follows

$$
\begin{gathered}
|a+\lambda+S|>2|a|, \\
|a-\lambda+S|<(\lambda-a+S) \operatorname{sign}(a+\lambda+S), \\
\sigma^{2}<\frac{4 S(\lambda-a)(\lambda+3 a+S)}{(\lambda-a+S)(\lambda+a+S)^{2}} .
\end{gathered}
$$

Similar for $\widehat{x}=\widehat{x}_{2}$ from (4.15) we obtain

$$
\begin{gathered}
|a-\lambda-S|+2|a|<|a+\lambda-S| \sqrt{1-\sigma^{2}}, \\
2|a|<|\lambda+a|-\sigma^{2} \frac{(\lambda+a-S)^{2}}{4 S}, \quad \lambda+a<0 .
\end{gathered}
$$




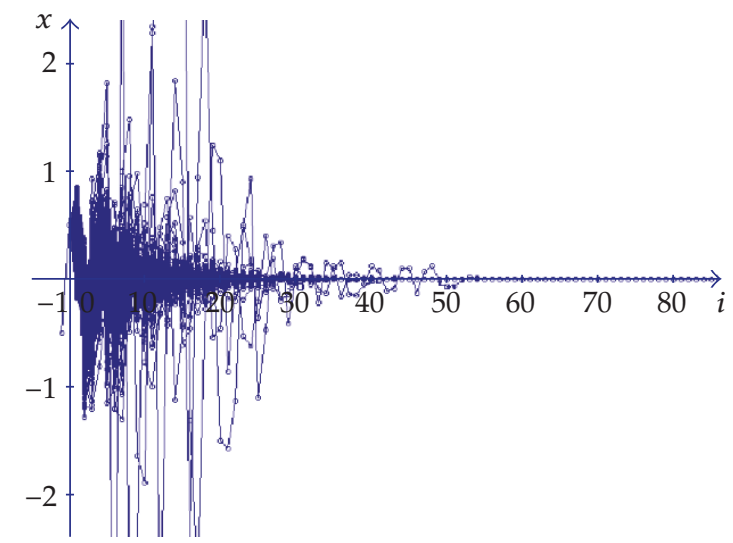

Figure 13: Stable equilibrium point $\widehat{x}_{2}=0$ for $\mu=0, \lambda=-2, a=1, \sigma=0.6$.

From (4.16) it follows

$$
\begin{gathered}
|a+\lambda-S|>2|a|, \\
|a-\lambda-S|<(\lambda-a-S) \operatorname{sign}(a+\lambda-S), \\
\sigma^{2}<\frac{4 S(a-\lambda)(\lambda+3 a-S)}{(\lambda-a-S)(\lambda+a-S)^{2}} .
\end{gathered}
$$

Put, for example, $\mu=0$. Then (4.13) has two equilibrium points: $\widehat{x}_{1}=a-\lambda, \widehat{x}_{2}=0$. From (4.15)-(4.16) it follows that the equilibrium point $\widehat{x}_{1}$ is unstable and the equilibrium point $\widehat{x}_{2}$ is stable in probability if and only if

$$
|\lambda|>\frac{|a|}{\sqrt{1-\sigma^{2}}}
$$

Note that for particular case $\mu=0, a=1, \lambda>0, \sigma=0$ in [35] it is shown that the equilibrium point $\widehat{x}_{2}$ is locally asymptotically stable if $\lambda>1$; and for particular case $\mu=0, a=-\alpha\langle 0, \lambda\rangle 0$, $\sigma=0$ in [18] it is shown that the equilibrium point $\widehat{x}_{2}$ is locally asymptotically stable if $\lambda>\alpha$. It is easy to see that both these conditions follow from (4.21).

Similar results can be obtained for the equation $x_{n+1}=\left(\mu-a x_{n}\right) /\left(\lambda+x_{n-1}\right)$ that was considered in [1].

In Figure 13 one thousand trajectories of (4.13) are shown for $\mu=0, \lambda=-2, a=1$, $\sigma=0.6, x_{-1}=-0.5, x_{0}=0.5$. In this case stability condition $(4.21)$ holds $(2>1.25)$ and therefore the equilibrium point $\hat{x}=0$ is stable: all trajectories go to zero. Putting $\sigma=0.9$, we obtain that stability condition $(4.21)$ does not hold $(2<2.29)$. Therefore, the equilibrium point $\hat{x}=0$ is unstable: in Figure 14 one can see that 1000 trajectories by the initial condition $x_{-1}=-0.1$, $x_{0}=0.1$ fill the whole space.

Example 4.4. Consider the equation

$$
x_{n+1}=\frac{p+x_{n-1}}{q x_{n}+x_{n-1}}+\sigma\left(x_{n}-\widehat{x}\right) \xi_{n+1}
$$




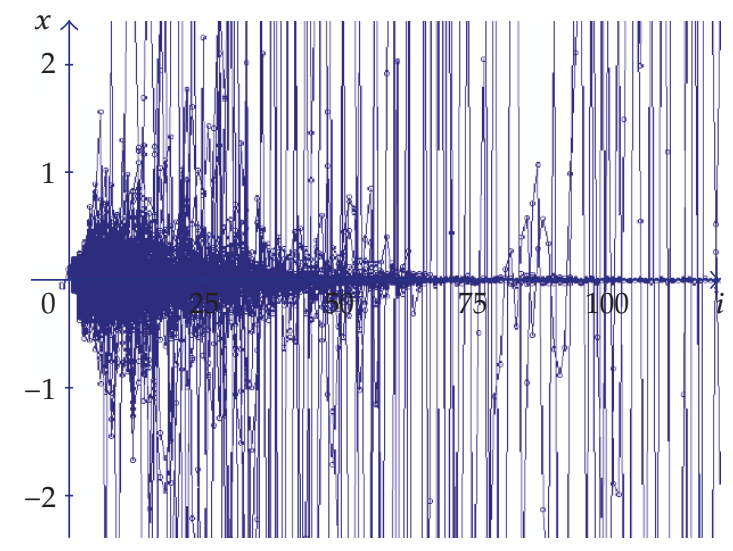

Figure 14: Unstable equilibrium point $\widehat{x}_{2}=0$ for $\mu=0, \lambda=-2, a=1, \sigma=0.9$.

that is a particular case of (3.10) with $\mu=p, \lambda=0, a_{0}=0, a_{1}=1, b_{0}=q, b_{1}=1$. As it follows from (1.4), (1.7) $-(1.9)$ by conditions $p(q+1)>-1 / 4, q \neq-1$, (4.22) has two equilibrium points

$$
\widehat{x}_{1}=\frac{1+S}{2(q+1)}, \quad \widehat{x}_{2}=\frac{1-S}{2(q+1)}, \quad S=\sqrt{1+4 p(q+1)} .
$$

From (3.11), (3.12) it follows that an equilibrium point $\widehat{x}$ of (4.22) is stable in probability if and only if

$$
\begin{gathered}
|1-\hat{x}|<|(q+1) \hat{x}|, \\
|q \hat{x}|<((2+q) \hat{x}-1) \operatorname{sign}((q+1) \widehat{x}), \\
\sigma^{2}<\frac{(1+q \widehat{x})(2 \widehat{x}-1)(2(q+1) \hat{x}-1)}{((2+q) \hat{x}-1)(q+1)^{2} \widehat{x}^{2}} .
\end{gathered}
$$

Substituting (4.23) into (4.24), we obtain stability conditions immediately in the terms of the parameters of considered equation (4.22): the equilibrium point $\widehat{x}_{1}$ is stable in probability if and only if

$$
p \in \begin{cases}\left(\frac{q-1}{4}, \infty\right), & q \geq 0, \\ \left(-\frac{1}{4(q+1)}, \frac{2}{q}+\frac{1}{q^{2}}\right), & q \in\left(-\frac{2}{3}, 0\right), \mid \sigma^{2}<\frac{4 S(S-q)((S+3) q+2)}{(S+1)^{2}(q+1)((q+2) S-q)},\end{cases}
$$

the equilibrium point $\widehat{x}_{2}$ is stable in probability if and only if

$$
p \in\left\{\begin{array}{ll}
\left(\frac{2}{q}+\frac{1}{q^{2}}, \infty\right), & q>0, \\
\left(\frac{q-1}{4}, \frac{2}{q}+\frac{1}{q^{2}}\right), & q<-2,
\end{array} \mid \sigma^{2}<\frac{4 S(S+q)((S-3) q-2)}{(S-1)^{2}(q+1)((q+2) S+q)}\right.
$$




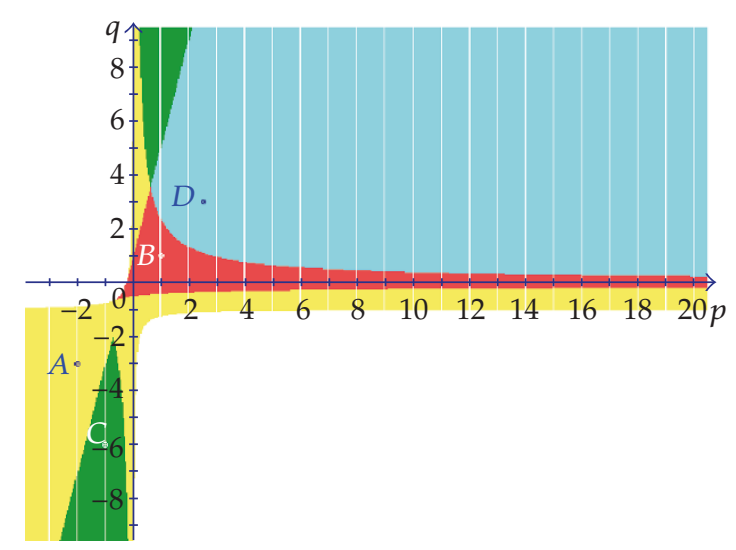

Figure 15: Stability regions, $\sigma=0$.

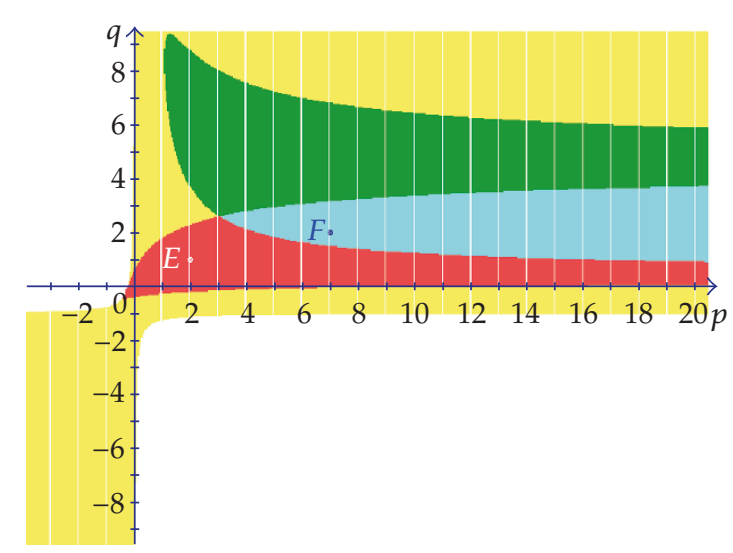

Figure 16: Stability regions, $\sigma=0.7$.

Note that in [24] equation (4.18) was considered with $\sigma=0$ and positive $p, q$. There it was shown that equilibrium point $\widehat{x}_{1}$ is locally asymptotically stable if and only if $4 p>q-1$ that is a part of conditions (4.25).

In Figure 15 the region where the points of equilibrium are absent (white region), the region where the both points of equilibrium $\widehat{x}_{1}$ and $\widehat{x}_{2}$ are there but unstable (yellow region), the region where the point of equilibrium $\widehat{x}_{1}$ is stable only (red region), the region where the point of equilibrium $\widehat{x}_{2}$ is stable only (green region) and the region where the both points of equilibrium $\widehat{x}_{1}$ and $\widehat{x}_{2}$ are stable (cyan region) are shown in the space of $(p, q)$. All regions are obtained via conditions (4.25), (4.26) for $\sigma=0$. In Figures 16 similar regions are shown for $\sigma=0.7$.

Consider the point $A$ (Figure 15) with $p=-2, q=-3$. In this point both equilibrium points $\widehat{x}_{1}=-1.281$ and $\widehat{x}_{2}=0.781$ are unstable. In Figure 17 two trajectories of solutions of (4.22) are shown with the initial conditions $x_{-1}=-1.28, x_{0}=-1.281$ and $x_{-1}=0.771, x_{0}=0.77$. In Figure 18 two trajectories of solutions of (4.22) with the initial conditions $x_{-1}=4, x_{0}=-3$ and $x_{-1}=-0.51, x_{0}=-0.5$ are shown in the point $B$ (Figure 15) with $p=q=1$. One can see that the equilibrium point $\widehat{x}_{1}=1$ is stable and the equilibrium point $\widehat{x}_{2}=-0.5$ is unstable. In the point $C$ (Figure 15) with $p=-1, q=-6$ the equilibrium point $\widehat{x}_{1}=-0.558$ is unstable and the equilibrium point $\widehat{x}_{2}=0.358$ is stable. Two corresponding trajectories of solutions are shown 


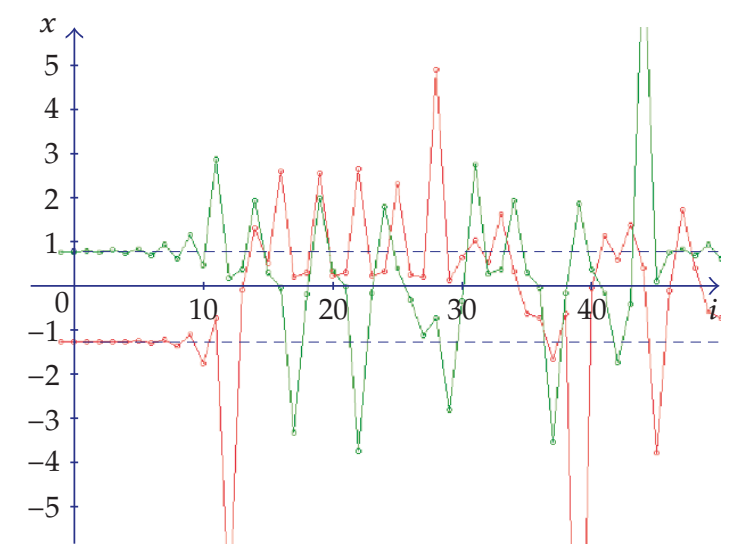

Figure 17: Unstable equilibrium points $\widehat{x}_{1}=-1.281$ and $\widehat{x}_{2}=0.781$ for $p=-2, q=-3$.

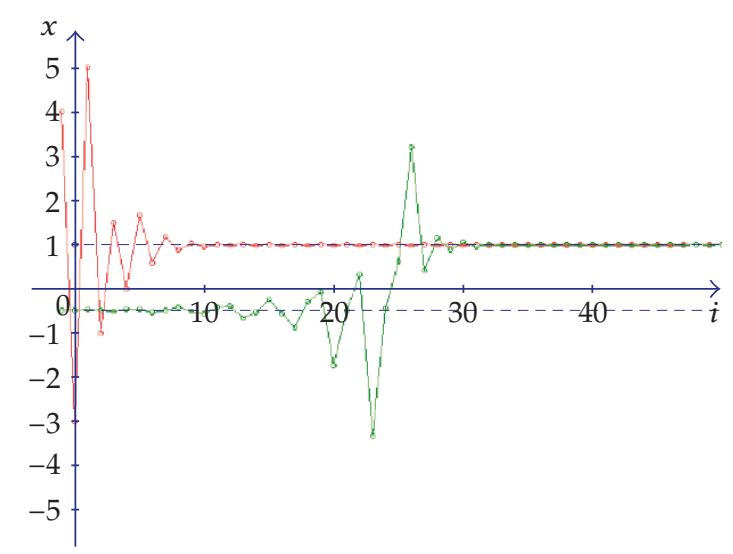

Figure 18: Stable equilibrium point $\widehat{x}_{1}=1$ and unstable $\widehat{x}_{2}=-0.5$ for $p=1, q=1$.

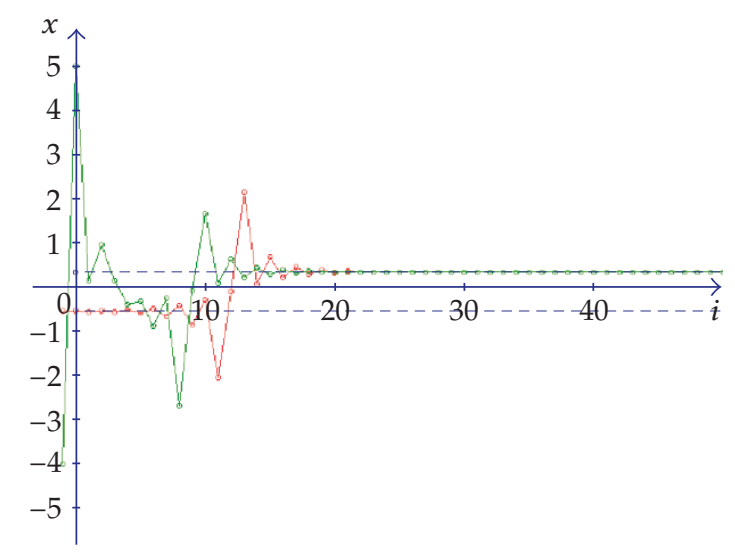

Figure 19: Unstable equilibrium point $\widehat{x}_{1}=-0.558$ and stable $\widehat{x}_{2}=0.358$ for $p=-1, q=-6$.

in Figure 19 with the initial conditions $x_{-1}=x_{0}=-0.55$ and $x_{-1}=-4, x_{0}=5$. In the point $D$ (Figure 15) with $p=2.5, q=3$ both equilibrium points $\hat{x}_{1}=0.925$ and $\hat{x}_{2}=-0.675$ are stable. Two corresponding trajectories of solutions are shown in Figure 20 with the initial conditions $x_{-1}=2.1, x_{0}=0.2$ and $x_{-1}=-0.2, x_{0}=-1.4$. 


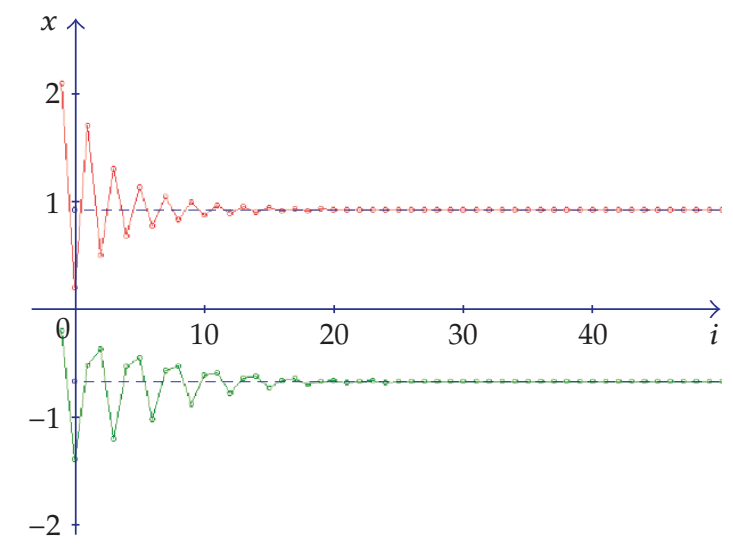

Figure 20: Stable equilibrium points $\widehat{x}_{1}=0.925$ and $\widehat{x}_{2}=-0.675$ for $p=2.5, q=3$.

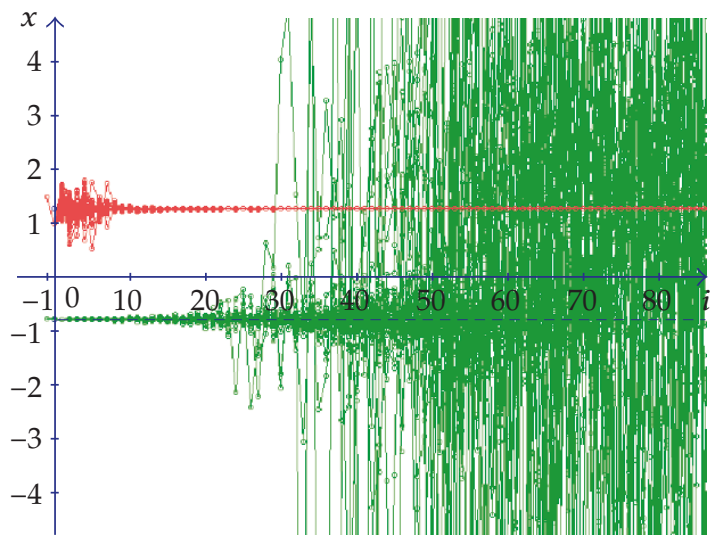

Figure 21: Stable equilibrium points $\widehat{x}_{1}=1.281$ and $\widehat{x}_{2}=-0.781$ for $p=2, q=1, \sigma=0.7$.

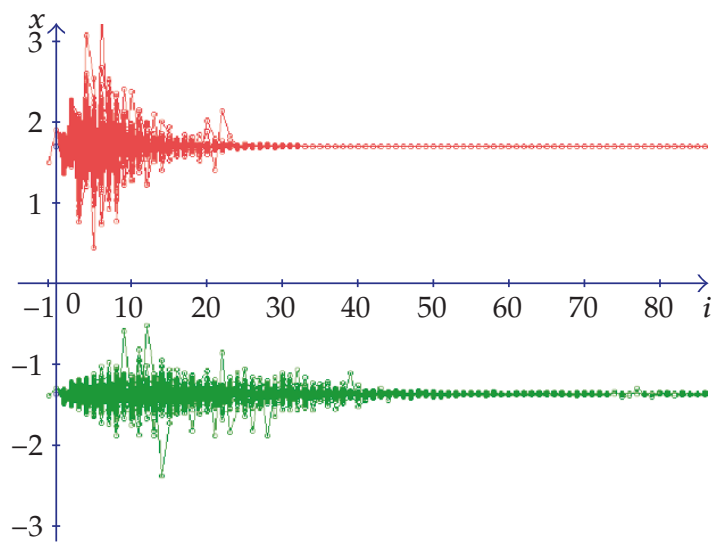

Figure 22: Stable equilibrium points $\widehat{x}_{1}=1.703$ and $\widehat{x}_{2}=-0.37$ for $p=7, q=2, \sigma=0.7$.

Consider the behavior of the equilibrium points of (4.22) by stochastic perturbations with $\sigma=0.7$. In Figure 21 trajectories of solutions are shown for $p=2, q=1$ (the point $E$ in Figure 16) with the initial conditions $x_{-1}=1.5, x_{0}=1$ and $x_{-1}=x_{0}=-0.78$. One can see that the equilibrium point $\widehat{x}_{1}=1.281$ (red trajectories) is stable and the equilibrium point 
$\widehat{x}_{2}=-0.781$ (green trajectories) is unstable. In Figure 22 trajectories of solutions are shown for $p=7, q=2$ (the point $F$ in Figure 16) with the initial conditions $x_{-1}=1.5, x_{0}=1.9$ and $x_{-1}=-1.4, x_{0}=-1.3$. In this case both equilibrium points $\hat{x}_{1}=1.703$ (red trajectories) and $\widehat{x}_{2}=-1.37$ (green trajectories) are stable.

\section{References}

[1] M. T. Aboutaleb, M. A. El-Sayed, and A. E. Hamza, "Stability of the recursive sequence $x_{n+1}=(\alpha-$ $\left.\beta x_{n}\right) /\left(\gamma+x_{n-1}\right)$," Journal of Mathematical Analysis and Applications, vol. 261, no. 1, pp. 126-133, 2001.

[2] R. M. Abu-Saris and R. DeVault, "Global stability of $y_{n+1}=A+\left(y_{n} / y_{n-k}\right)$," Applied Mathematics Letters, vol. 16, no. 2, pp. 173-178, 2003.

[3] A. M. Amleh, E. A. Grove, G. Ladas, and D. A. Georgiou, "On the recursive sequence $x_{n+1}=\alpha+$ $\left(x_{n-1} / x_{n}\right), "$ Journal of Mathematical Analysis and Applications, vol. 233, no. 2, pp. 790-798, 1999.

[4] K. S. Berenhaut, J. D. Foley, and S. Stević, "Quantitative bounds for the recursive sequence $y_{n+1}=$ $A+\left(y_{n} / y_{n-k}\right)$," Applied Mathematics Letters, vol. 19, no. 9, pp. 983-989, 2006.

[5] K. S. Berenhaut, J. D. Foley, and S. Stević, "The global attractivity of the rational difference equation $y_{n}=1+\left(y_{n-k} / y_{n-m}\right), "$ Proceedings of the American Mathematical Society, vol. 135, no. 4, pp. 1133-1140, 2007.

[6] K. S. Berenhaut and S. Stević, "The difference equation $x_{n+1}=\alpha+\left(x_{n-k} / \sum_{i=0}^{k-1} c_{i} x_{n-i}\right)$ has solutions converging to zero," Journal of Mathematical Analysis and Applications, vol. 326, no. 2, pp. 1466-1471, 2007.

[7] E. Camouzis, E. Chatterjee, and G. Ladas, "On the dynamics of $x_{n+1}=\left(\delta x_{n-2}+x_{n-3}\right) /\left(A+x_{n-3}\right)$," Journal of Mathematical Analysis and Applications, vol. 331, no. 1, pp. 230-239, 2007.

[8] E. Camouzis, G. Ladas, and H. D. Voulov, "On the dynamics of $x_{n+1}=\left(\alpha+\gamma x_{n-1}+\delta x_{n-2}\right) /\left(A+x_{n-2}\right)$," Journal of Difference Equations and Applications, vol. 9, no. 8, pp. 731-738, 2003.

[9] E. Camouzis and G. Papaschinopoulos, "Global asymptotic behavior of positive solutions on the system of rational difference equations $x_{n+1}=1+x_{n} / y_{n-m}, y_{n+1}=1+y_{n} / x_{n-m}$, , Applied Mathematics Letters, vol. 17, no. 6, pp. 733-737, 2004.

[10] C. Çinar, "On the difference equation $x_{n+1}=x_{n-1} /\left(-1+x_{n} x_{n-1}\right)$, " Applied Mathematics and Computation, vol. 158, no. 3, pp. 813-816, 2004.

[11] C. Çinar, "On the positive solutions of the difference equation $x_{n+1}=\alpha x_{n-1} /\left(1+b x_{n} x_{n-1}\right), "$ Applied Mathematics and Computation, vol. 156, no. 2, pp. 587-590, 2004.

[12] C. Çinar, "On the positive solutions of the difference equation system $x_{n+1}=1 / y_{n}, y_{n+1}=$ $y_{n} / x_{n-1} y_{n-1}, "$ Applied Mathematics and Computation, vol. 158, no. 2, pp. 303-305, 2004.

[13] C. Çinar, "On the positive solutions of the difference equation $x_{n+1}=x_{n-1} /\left(1+x_{n} x_{n-1}\right)$," Applied Mathematics and Computation, vol. 150, no. 1, pp. 21-24, 2004.

[14] D. Clark and M. R. S. Kulenović, "A coupled system of rational difference equations," Computers $\mathcal{E}$ Mathematics with Applications, vol. 43, no. 6-7, pp. 849-867, 2002.

[15] C. A. Clark, M. R. S. Kulenović, and J. F. Selgrade, "On a system of rational difference equations," Journal of Difference Equations and Applications, vol. 11, no. 7, pp. 565-580, 2005.

[16] R. DeVault, C. Kent, and W. Kosmala, "On the recursive sequence $x_{n+1}=p+\left(x_{n-k} / x_{n}\right)$," Journal of Difference Equations and Applications, vol. 9, no. 8, pp. 721-730, 2003.

[17] E. M. Elabbasy, H. El-Metwally, and E. M. Elsayed, "On the difference equation $x_{n+1}=a x_{n}-b x_{n} /\left(c x_{n}-\right.$ $\left.d x_{n-1}\right), "$ Advances in Difference Equations, vol. 2006, Article ID 82579, 10 pages, 2006.

[18] H. M. El-Owaidy, A. M. Ahmed, and M. S. Mousa, "On the recursive sequences $x_{n+1}=-\alpha x_{n-1} /(\beta+$ $\left.x_{n}\right)$," Applied Mathematics and Computation, vol. 145, no. 2-3, pp. 747-753, 2003.

[19] C. H. Gibbons, M. R. S. Kulenovic, and G. Ladas, "On the recursive sequence $x_{n+1}=\left(\alpha+\beta x_{n-1}\right) /(\gamma+$ $\left.x_{n}\right), "$ Mathematical Sciences Research Hot-Line, vol. 4, no. 2, pp. 1-11, 2000.

[20] C. H. Gibbons, M. R. S. Kulenović, G. Ladas, and H. D. Voulov, "On the trichotomy character of $x_{n+1}=\left(\alpha+\beta x_{n}+\gamma x_{n-1}\right) /\left(A+x_{n}\right)$," Journal of Difference Equations and Applications, vol. 8, no. 1, pp. 75-92, 2002.

[21] E. A. Grove, G. Ladas, L. C. McGrath, and C. T. Teixeira, "Existence and behavior of solutions of a rational system," Communications on Applied Nonlinear Analysis, vol. 8, no. 1, pp. 1-25, 2001. 
[22] L. Gutnik and S. Stević, "On the behaviour of the solutions of a second-order difference equation," Discrete Dynamics in Nature and Society, vol. 2007, Article ID 27562, 14 pages, 2007.

[23] A. E. Hamza, "On the recursive sequence $x_{n+1}=\alpha+\left(x_{n-1} / x_{n}\right)$," Journal of Mathematical Analysis and Applications, vol. 322, no. 2, pp. 668-674, 2006.

[24] W. A. Kosmala, M. R. S. Kulenović, G. Ladas, and C. T. Teixeira, "On the recursive sequence $y_{n+1}=$ $\left(p+y_{n-1}\right) /\left(q y_{n}+y_{n-1}\right), "$ Journal of Mathematical Analysis and Applications, vol. 251, no. 2, pp. 571-586, 2000.

[25] M. R. S. Kulenović and G. Ladas, Dynamics of Second Order Rational Difference Equations: With Open Problems and Conjectures, Chapman \& Hall/CRC, Boca Raton, Fla, USA, 2001.

[26] M. R. S. Kulenović and M. Nurkanović, "Asymptotic behavior of a competitive system of linear fractional difference equations," Advances in Difference Equations, vol. 2006, Article ID 19756, 13 pages, 2006.

[27] M. R. S. Kulenović and M. Nurkanović, "Asymptotic behavior of a two dimensional linear fractional system of difference equations," Radovi Matematički, vol. 11, no. 1, pp. 59-78, 2002.

[28] M. R. S. Kulenović and M. Nurkanović, "Asymptotic behavior of a system of linear fractional difference equations," Journal of Inequalities and Applications, vol. 2005, no. 2, pp. 127-143, 2005.

[29] X. Li, "Global behavior for a fourth-order rational difference equation," Journal of Mathematical Analysis and Applications, vol. 312, no. 2, pp. 555-563, 2005.

[30] X. Li, "Qualitative properties for a fourth-order rational difference equation," Journal of Mathematical Analysis and Applications, vol. 311, no. 1, pp. 103-111, 2005.

[31] G. Papaschinopoulos and C. J. Schinas, "On the system of two nonlinear difference equations $x_{n+1}=$ $A+x_{n-1} / y_{n}, y_{n+1}=A+y_{n-1} / x_{n}$," International Journal of Mathematics and Mathematical Sciences, vol. 23, no. 12 , pp. 839-848, 2000.

[32] S. Stević, "On the recursive sequence $x_{n}=1+\left(\sum_{i=1}^{k} \alpha_{i} x_{n-p_{i}} / \sum_{j=1}^{m} \beta_{j} x_{n-q_{j}}\right)$," Discrete Dynamics in Nature and Society, vol. 2007, Article ID 39404, 7 pages, 2007.

[33] T. Sun and H. Xi, "On the system of rational difference equations $x_{n+1}=f\left(x_{n-q}, y_{n-s}\right), y_{n+1}=$ $f\left(y_{n-l}, y_{n-p}\right), "$ Advances in Difference Equations, vol. 2006, Article ID 51520, 8 pages, 2006.

[34] T. Sun, H. Xi, and L. Hong, "On the system of rational difference equations $x_{n+1}=f\left(x_{n}, y_{n-k}\right), y_{n+1}=$ $f\left(y_{n}, x_{n-k}\right), "$ Advances in Difference Equations, vol. 2006, Article ID 16949, 7 pages, 2006.

[35] T. Sun, H. Xi, and H. Wu, "On boundedness of the solutions of the difference equation $x_{n+1}=x_{n-1} /(p+$ $\left.x_{n}\right)$," Discrete Dynamics in Nature and Society, vol. 2006, Article ID 20652, 7 pages, 2006.

[36] H. Xi and T. Sun, "Global behavior of a higher-order rational difference equation," Advances in Difference Equations, vol. 2006, Article ID 27637, 7 pages, 2006.

[37] X.-X. Yan, W.-T. Li, and Z. Zhao, "On the recursive sequence $x_{n+1}=\alpha-x_{n} / x_{n-1}$," Journal of Applied Mathematics \& Computing, vol. 17, no. 1-2, pp. 269-282, 2005.

[38] X. Yang, "On the system of rational difference equations $x_{n}=A+y_{n-1} / x_{n-p} y_{n-q}, y_{n}=A+$ $x_{n-1} / x_{n-r} y_{n-s}, "$ Journal of Mathematical Analysis and Applications, vol. 307, no. 1, pp. 305-311, 2005.

[39] E. Beretta, V. Kolmanovskii, and L. Shaikhet, "Stability of epidemic model with time delays influenced by stochastic perturbations," Mathematics and Computers in Simulation, vol. 45, no. 3-4, pp. 269-277, 1998.

[40] L. Shaikhet, "Stability of predator-prey model with aftereffect by stochastic perturbation," Stability and Control: Theory and Applications, vol. 1, no. 1, pp. 3-13, 1998.

[41] M. Bandyopadhyay and J. Chattopadhyay, "Ratio-dependent predator-prey model: effect of environmental fluctuation and stability," Nonlinearity, vol. 18, no. 2, pp. 913-936, 2005.

[42] N. Bradul and L. Shaikhet, "Stability of the positive point of equilibrium of Nicholson's blowflies equation with stochastic perturbations: numerical analysis," Discrete Dynamics in Nature and Society, vol. 2007, Article ID 92959, 25 pages, 2007.

[43] M. Carletti, "On the stability properties of a stochastic model for phage-bacteria interaction in open marine environment," Mathematical Biosciences, vol. 175, no. 2, pp. 117-131, 2002.

[44] V. Kolmanovskii and L. Shaikhet, "General method of Lyapunov functionals construction for stability investigation of stochastic difference equations," in Dynamical Systems and Applications, vol. 4 of World Scientific Series in Applicable Analysis, pp. 397-439, World Science, River Edge, NJ, USA, 1995.

[45] V. Kolmanovskii and L. Shaikhet, "Construction of Lyapunov functionals for stochastic hereditary systems: a survey of some recent results," Mathematical and Computer Modelling, vol. 36, no. 6, pp. 691-716, 2002. 
[46] L. Shaikhet, "Necessary and sufficient conditions of asymptotic mean square stability for stochastic linear difference equations," Applied Mathematics Letters, vol. 10, no. 3, pp. 111-115, 1997.

[47] B. Paternoster and L. Shaikhet, "About stability of nonlinear stochastic difference equations," Applied Mathematics Letters, vol. 13, no. 5, pp. 27-32, 2000.

[48] L. Shaikhet, "Stability in probability of nonlinear stochastic systems with delay," Matematicheskie Zametki, vol. 57, no. 1, pp. 142-146, 1995 (Russian).

[49] L. Shaikhet, "Stability in probability of nonlinear stochastic hereditary systems," Dynamic Systems and Applications, vol. 4, no. 2, pp. 199-204, 1995. 